\title{
Superior vena cava syndrome
}

\begin{abstract}
The superior vena cava (SVC) located in anterior mediastinum, Carries blood from the head, neck, upper extremities and upper chest. Rodeada by rigid structures and compress the upper originate vena cava syndrome (SVCS). The may be due to obstruction external compression (benign or malignant), Fibrosis or thrombosis. Venous hypertension produces head, neck and upper extremities azygos and deriving flow systems. Symptoms depend on the speed of obstruction and location is quick. If symptoms are intense. More often slow installation. Initial symptoms, Drowsiness, tinnitus, dizziness, cervical increased diameter. If Endures, cyanosis of the face, neck, upper limbs, altered state of consciousness.
\end{abstract}

Examination: Plethora jugular, thoracic collateral circulation. The diagnosis clinical, in tele-ray, mediastinal masses, pleural effusion, lobar collapse, or cardiomegaly. The TAC defines the anatomy of mediastinal nodes and VCS, the site of obstruction and Provides guidance for needle biopsy, bronchoscopy or mediastinoscopy. Contrast venography, ultrasound and MRI are used to determine the site and nature of the obstruction. The treatment of malignant obstruction histological diagnosis by sputum requires cytology, biopsy or lymph.

Treatment: General Measures and the underlying cause, if chemotherapy or radiotherapy neoplastic in thrombosis, thrombectomy plasminogen activator (TPA), streptokinase or urokinase. Aangioplastia intraluminal stent, bypass in malignant etiology no chemotherapy and radiotherapy with improvement.

Driving urgent: Patients with cerebral edema, airway obstruction due to compression of the trachea, for airway edema, or cardiac output decreased in venous return. Mortality depends on the underlying cause.

Keywords: superior vena cava syndrome, mediastinal masses, thrombosis
Volume II Issue 6 - 2018

\author{
Juan Manuel Cortes Ramírez,' Juan Manuel \\ de Jesús Cortes de la Torre, ${ }^{2}$ Raúl Arturo \\ Cortes de la Torre, ${ }^{3}$ Alfredo Salazar de \\ Santiago,' Oscar Octavio Ramos Castelo \\ Francisco Patiño Diego Lopez ${ }^{3}$ David \\ Ricardo Ramirez Carranza' \\ 'Area of Health Sciences, Autonomous University of Zacatecas, \\ Mexico \\ ${ }^{2}$ Chair, Department of Cardiology, National Institute of \\ Cardiology, Mexico \\ ${ }^{3}$ Ignacio Morones School of Medicine, Mexico
}

\begin{abstract}
Correspondence: Juan Manuel Cortes Ramírez, Area of Health Sciences, Autonomous University of Zacatecas, Mexico, Emaildr.cortesramerez@gmail.com
\end{abstract}

Received: October 25, 2018 | Published: November 15, 2018

\section{Introduction}

The superior vena cava (VCS) is located in the anterior mediastinum, he carries venous blood from head, neck, upper extremities and upper chest to the right heart. Surrounded by more rigid structures: sternum, trachea and right main bronchus, aorta, pulmonary artery and enveloped by parahilar and paratracheal lymph nodes, ${ }^{1}$ causing a distensible place and when compressed by any space-occupying lesion gives SVCS..$^{2,3}$ Obstruction may be caused by external compression, neoplasia, mediastinal lymphadenopathy, ${ }^{4}$ fibrosis or thrombosis, leading venous hypertension of the head, neck and upper extremities. ${ }^{2}$ The venous flow is then derived Azygos and its collateral systems (internal mammary veins, paraspinal esophageal veins and venous network). Subcutaneous veins are the most important roads and engorgement in the neck and chest is typical causes of SVCS. Malignant origin-bronchogenic Carcinoma-small cell, squamous, adenocarcinoma, and grandes. Hodgkin lymphoma cells and rarely Hodgkin. Breast carcinoma, thymoma and tumor cells metastatic tumors germinales. breast, testis colon. Benign causes-Bocio endotorácico, Sarcoidosis, thrombosis associated with catheters pacemaker or-reservoirs or chemotherapy, as well as temporary and permanent catheters in hemodialysis patients nefrópatas, Behcet,Mediastinitis fibrous (idiopathic or associated Histoplasmosis), Tuberculosis and syphilis (rare). ${ }^{2}$

Dependending symptoms of the rapidity of the establishment of obstruction and location. ${ }^{2}$ If it does quickly the symptoms will be more intense. It is more frequent slow installation According the site of obstruction, you can be found as the above input Azygos, the syndrome is less pronounced, Azygos distends quickly and accommodates the blood and causes lower venous pressure head, arms and chest. ${ }^{2}$ Blood returns to the VCS by internal and costoaxilares mammary veins, so that the venous circulation is not noticeable on the chest wall. It present distended jugular vein. ${ }^{5}$ Below the entrance of the azygos vein-Symptoms and signs are more flowery, the blood returns to the heart via the upper abdominal veins and inferior vena cava, ${ }^{2}$ large thoracoabdominal collateral circulation and lower limb edema and ascites. ${ }^{5}$

\section{The initial symptoms}

Headache increases with decubitus, drowsiness, tinnitus, dizziness, increased cervical diameter. ${ }^{5}$ If thrombosis can present pain in the jaw, neck and shoulder, without collateral circulation. ${ }^{2}$ If the compression lasts cyanosis of skin, mucous face, neck, upper limbs, conjunctival hemorrhage, edema esclavina (face, neck and upper chest) predominance morning and exophthalmos time and macroglossia will develop. In severe cases disturbances of consciousness ${ }^{4}$

1. Other symptoms are hoarseness, nasal congestion, epistaxis, hemoptysis, dysphagia, pain, dizziness, syncope and lethargy. ${ }^{3}$

2. At physical examination: thoracic collateral circulation, varying plethora jugular. It may appear Horner Syndrome compression of the sympathetic chain in the mediastinum ${ }^{4}$ 
3. 'The diagnosis is clinical.

4. Radiografía chest to look for mediastinal masses, pleural effusion, lobar collapse, or cardiomegaly. ${ }^{5}$

$90 \%$ shows an alteration. $75 \%$ is a mass in the right upper mediastinum and $50 \%$ combined with a lung injury or hilar lymphadenopathy. $25 \%$ there is pleural effusion, usually on the right side. $^{2}$

5. The computed tomography (CT), it is defined the anatomy of mediastinal nodes affected. ${ }^{6}$ Of choice to evaluate the anatomy of the mediastinum and the structure of the VCS. Locates the site of obstruction and serves as a guide for biopsy by mediastinoscopy, bronchoscopy or aspiration. ${ }^{4}$

6. La contrast venography, ultrasound and magnetic resonance imaging to determine the site and nature of the obstruction. ${ }^{6}$ The latter has no advantage over the TAC. ${ }^{3}$

7. In patients with known malignancy is not necessary to conduct further diagnostic techniques. ${ }^{3}$

8. The treatment of malignant obstruction depends on the histology of the tumor, should make a histological diagnosis ${ }^{6}$ by sputum cytology, biopsy of the tumor mass or lymph CT guided puncturing susceptible. $^{4}$

9. When there is no history of malignancy should be ruled benign causes. $^{3}$

\section{Treatment}

\section{General measures}

Avoid lying, Rest in semi-sitting position, Oxygen, Diuretics, lowsodium diet, Corticosteroids ${ }^{1}$

\section{Emergency management}

In patients with cerebral edema, obstruction of the airway by compression of the trachea or airway edema, decreased cardiac output or by decreased venous return. ${ }^{4}$ The underlying cause neoplasic, chemotherapy offers improvement over $65 \%$ of the SVCS are produced by highly chemosensitive and curable neoplasms. ${ }^{1}$ The treatment of choice of primary mediastinal B-cell lymphoma is chemotherapy, radiotherapy associating. ${ }^{2}$ The choice therapy is not sensitive tumors to chemotherapy. ${ }^{2}$ In patients with a thrombus in the VCS, thrombectomy with or without tissue plasminogen activator (TPA) or other thrombolytic, streptokinase or urokinase. ${ }^{6}$ Another treatment intraluminal angioplasty SX stents, bypass surgery in malignant etiology that have not improved with chemo- and radiotherapy. ${ }^{1}$

\section{Complications}

The life-threatening complication when there is a mass in the upper mediastinum is the obstruction of the trachea and requires urgent treatment. Mortality does not depend on the obstruction but the underlying cause. ${ }^{3}$

\section{Clinical case}

Male 55 years of age, genetic burden to you, dm, heart disease. Tab up to 6 months 5 cigarettes a day for 15 años. PA Starts 4 months myalgia and arthralgia, headache with decubitus, dizziness, dry cough without predominance of time, wheezy, hyperoxia, tired, exhausted, insomnia, lateropulsion, dorsalgia disfonía ago , dyspnea small efforts, night sweats, back pain, facial edema, lower limb, abominal, upper limb and neck EFTA 90/60, FC 120X MIN, facial edema and neck, even sitting jugular plethora, thoracic venous network, adenomegalia bilateral neck and underarm, edema and abdominal wall members inferiores (Figure 1). He underwent chest CT lung tumor reporting based region right evil-looking, mediastinal tumor with calcifications and data invasion of pericardium and conditioning sves superior vena cava, mediastinal lymphadenopathy of bilateral hilar groups prevascular (Figure 2) (Figure 3).

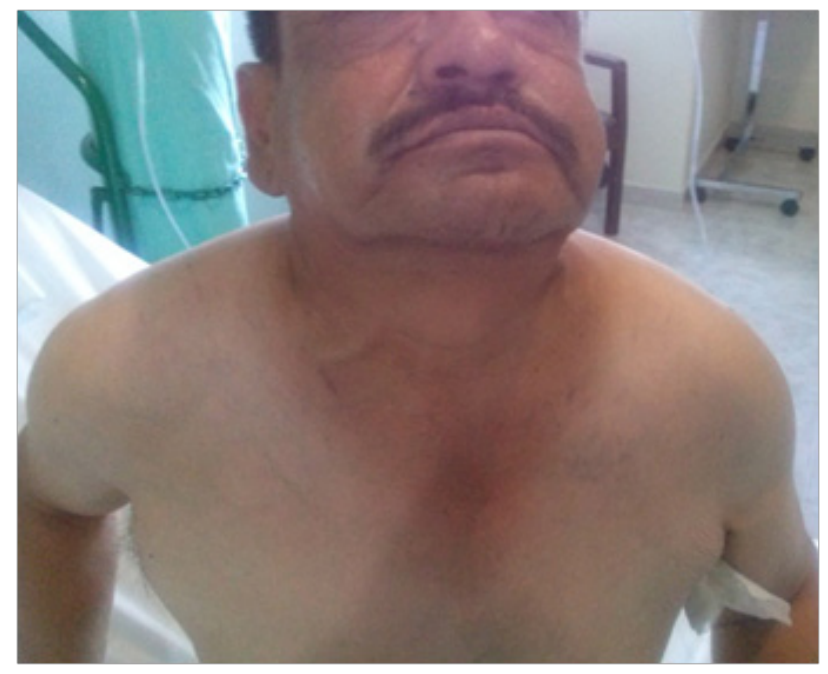

Figure I Clinical examination.

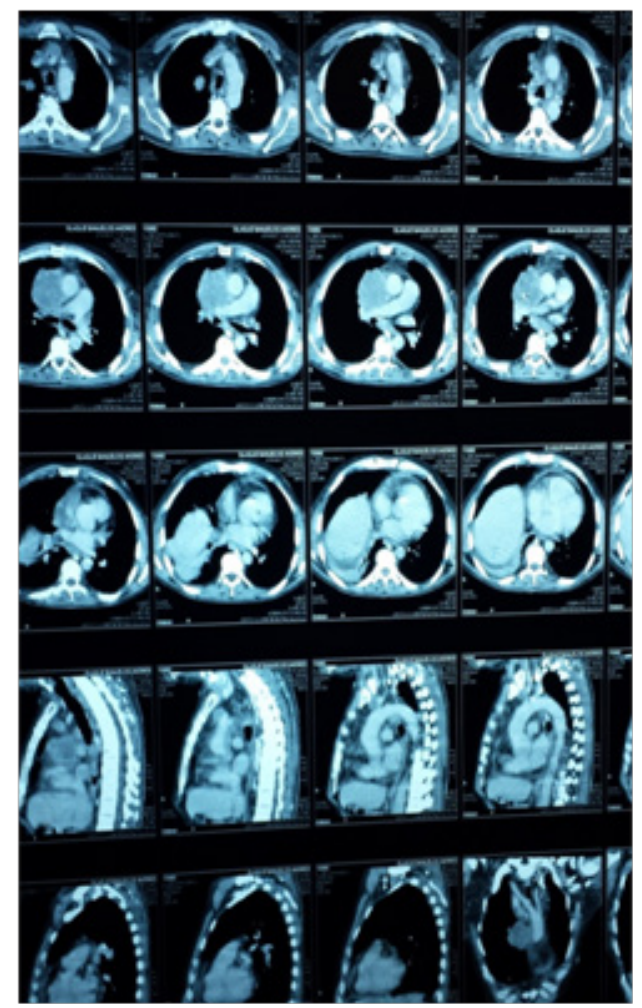

Figure 2 Report of CT lung tumor. 


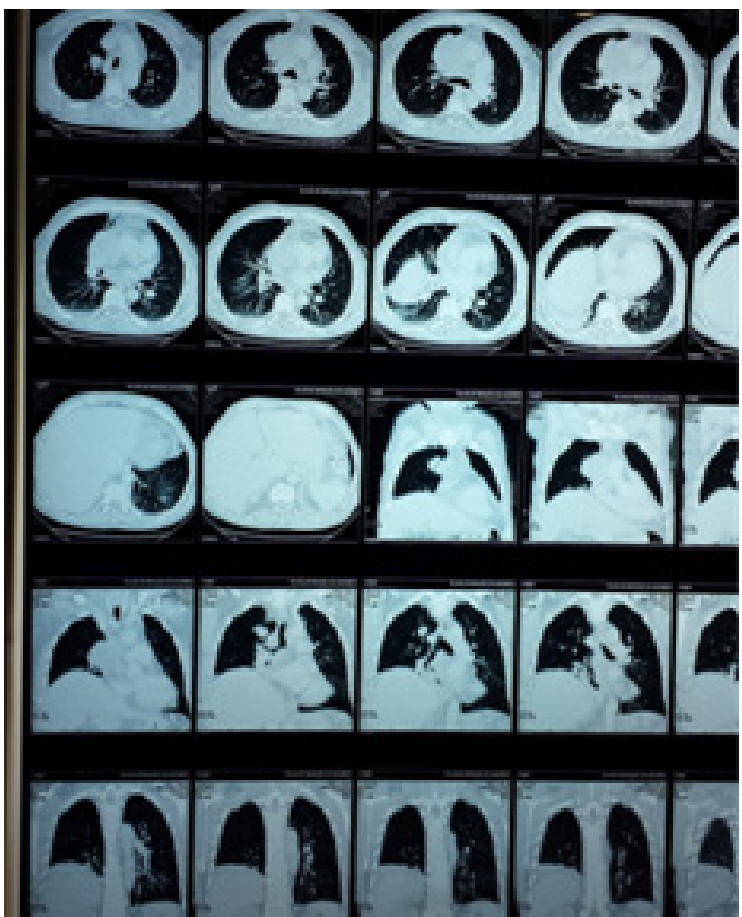

Figure 3 Report of CT lung tumor.

\section{Conclusion}

SVCS is a medical emergency, its importance lies in finding the underlying cause, often neoplasias. The installation depends on the location of the mass, speed of installation, therapy.Improper success of endovascular treatment is an effective and safe procedure, minimally invasive and low morbidity and mortality provide rapid improvement in the quality of life of patients compressive and symptoms.

\section{Acknowledgements}

None.

\section{Conflict of interest}

Author declares that there is no conflicto of interest.

\section{References}

1. Shims J, Lianes P, Cortés Funes H. Heart and neoplasias: Update. Heart diseases cardiac origin. Spanish Journal of Cardiology. 1998;51(4).

2. Zomeño Rodriguez M. Superior vena cava syndrome. Spain Med. 2000.

3. Gucalp R, Dutcher J. Oncologic emergency. In: Isselbacher KJ, Braunwaid E, Wilson JD, Martin JB, Fauci AS, Kasper DL, Harrison, editors. Principles of Internal Medicine. 15th edition. Mexico: Mc GrawHill publishers SA de CU, 2002;1:760-769.

4. Vargas CA, Martinez I. Superior vena cava syndrome. Guide for emergency management. Pan American Federation of Associations of Colleges and Schools of Medicine, 2004;19:1503-1506.

5. Arribalzaga EB. Mediastinal syndrome. In: Ferraina P, Oria, editors. Michans Surgery 5th Edition. Argentina: Editorial El Ateneo; 2003:372-373.

6. Quade G. Superior vena cava syndrome. Med News National Cancer Institute. 2002. 